Artículo de investigación

Cuestiones de Filosofía

ISSN: 0123-50-95

E-ISSN: 2389-9441

Vol. 5 - Nº 24 Enero- junio, año 2019

pp. 21-48

\title{
Una propuesta de orientaciones pedagógicas desde la filosofía de Nuestramérica para la enseñanza de la filosofía en Colombia.
}

\section{A proposal of pedagogic orientations from the "Nuestramerica" philosophy for the teaching of philosophy in Colombia.}

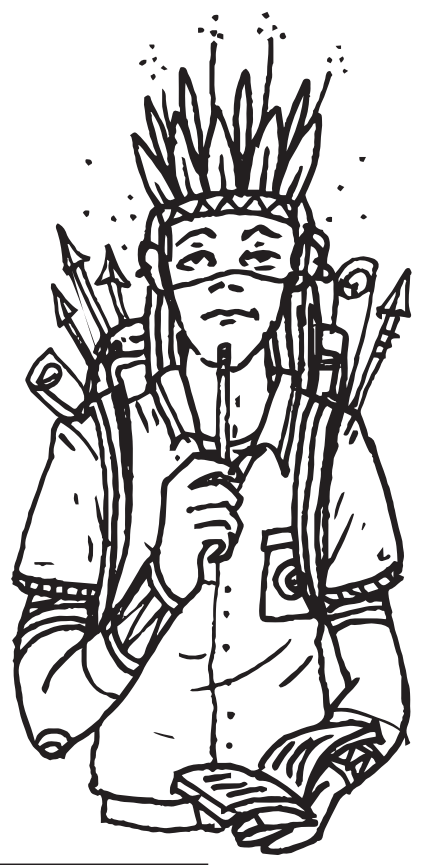

Diana Melisa Paredes Oviedo² Universidad de Antioquia, Colombia Andrés Carmona-Cardona ${ }^{3}$ Institución Educativa Josefina Muñoz González, Colombia

1 Este artículo de reflexión se deriva del trabajo de investigación, de la Maestria en Educación de la Universidad de Antioquia, delestudiante Andrés Carmona-Cardona, titulado Orientaciones Pedagógicas para la Filosofía desde Nuestramérica, dirigido por la profesora Diana Melisa Paredes Oviedo, quien está adscrita al grupo de investigación Sobre Formación y Antropología Pedagógica e Histórica (Formaph), de la Facultad de Educación de la Universidad de Antioquia.

2 Magíster en Educación y Magíster en Filosofía de la Universidad de Antioquia. Correo electrónico: diana.paredes@udea.edu.co.

3 Licenciado en Filosofia y Candidato a Magister en Educación por la Universidad de Antioquia. Correo electrónico: andresfurcas@gmail.com. 


\section{Resumen:}

La enseñanza de la filosofia, en el nivel de la educación media en Colombia, ha estado marcada por profundas transformaciones a partir de la década de 1990. Su orientación ha sido hacia un marcado tratamiento de la filosofía occidental, desde una perspectiva histórico-lineal, como único relato y a su apropiación a través de la ontología, la cosmología y la epistemología, o por preguntas de estética, ética o cosmología. También por la adopción de un enfoque de educación por competencias básicas y específicas, como líneas fuertes en el proceso de formación de los estudiantes colombianos, que paulatinamente ha ido desdibujando la especificidad de lo filosófico y encaminando los procesos formativos en esta área hacia la formación en lectura crítica. Este trabajo apunta, en primer lugar, a una revisión de esos cambios y, en segundo lugar, a la presentación de una propuesta de Orientaciones Pedagógicas para la Enseñanza de la Filosofía desde la filosofía de Nuestramérica, que recoge una perspectiva decolonial y crítica de la formación como alternativas a la necesidad de una formación filosófica situada, junto con la ampliación del concepto de competencias desde la perspectiva del desarrollo humano.

Palabras Clave: Enseñanza de la Filosofía, Enseñanza Secundaria, Filosofía de la Educación.

\section{Abstract:}

The teaching of philosophy, in the middle education level in Colombia, has been marked by deep transformations since the 1990s. Its orientation has been aligned with western philosophy, from a lineal-historical perspective, as a single story and its appropriation through the ontology, cosmology, and epistemology, or by questions about esthetics, ethic, or cosmology. Likewise, by the adoption of a focus for education toward the basic and specific competencies, as strong guidelines in the formation process of the Colombian students, who slowly have blurred the specificity of the philosophical aspect and, pointing the formative processes in this area towards the formation in critical reading. This work aims to, in the first place, a review of those transformations and second, to the presentation of a proposal of Pedagogical orientations for teaching philosophy from "Nuestramerica" philosophy. This philosophy gathers a decolonial and critical perspective of the formation as an 
alternative to the need of a philosophical formation located near of the broadening development of the concept of competences from a human development perspective.

Key-word: Teaching of Philosophy, Secondary Education, Philosophy of Education.

\section{Une proposition d'orientations pédagogiques à partir de la philosophie de « Nuestramerica » pour l'enseignement de la philosophie en Colombie}

\section{Résumé :}

L'enseignement de la philosophie dans le niveau d'éducation secondaire en Colombie a été marquée par de profondes transformations dès le fil des années 90. Son orientation a été guidée vers un traitement de la philosophie occidentale, d'un point de vue historique-linéaire, comme l'unique concept grâce à sa propre redéfinition à travers de l'ontologie, de la cosmologie, et de l'épistémologie ou par des questions esthétiques, éthiques et cosmologiques ; en plus, par l'adoption d'une approche d'éducation liée au développement de compétences basiques et spécifiques, comme les pours dans le processus de formation des apprenants colombiens ; laquelle peu à peu a commencé a détourner l'spécifié de ce qui est philosophique en transformant son essence dans une formation de lecture critique. D'abord, ce travail est orienté vers une révision de ceux changements et à la présentation d'une proposition d'Orientations Pédagogiques pour l'Enseignement de la Philosophie à partie de la philosophie « Nuestramérica », qui prends en soi une perspective décoloniale et critique de la formation, comme des alternatives au besoin d'une vraie formation philosophique, toujours avec l'élargissement du concept de compétences dès la perspective du développement humain.

Mots-clés : Enseignement de la philosophie, enseignement secondaire, philosophie de l'éducation. 


\section{Uma proposta de orientação pedagógicas desde a filosofia de "Nuestramerica" para o ensino da filosofia na Colômbia}

\section{Resumo:}

O ensino da filosofia, no nivel de educação media na Colômbia, tem marcas de profundas transformações a partir da década de 1990. Sua orientação tem sido marcada pelo tratamento da filosofia ocidental, desde uma perspectiva históricolineal, como único relato e sua apropriação a traves da ontologia, cosmologia e epistemologia, ou por perguntas de estética, ética e cosmologia. Também pela adopção de um enfoque de educação por competências básicas e especificas, como líneas fortes no processo de formação dos estudantes colombianos que, paulatinamente, foram desenhando a especificidade do filosófico e encaminhando os processos formativos nesta área para a formação de leitura critica. Este trabalho propor, em primeiro lugar, uma revisão dessas mudanças e, no segundo lugar, à apresentação da proposta sobre Orientações Pedagógicas para o Ensino da Filosofia desde a filosofia de "Nuestramérica" que, recolhe uma perspectiva decolonial e critica da formação como alternativas na necessidade de uma formação filosófica situada, junto com a ampliação do conceito de competências desde a perspectiva do desenvolvimento humano.

Palavras Chave: Ensino da filosofia, Ensino Meio, Filosofia da educação. 


\section{Generalidades sobre la enseñanza de la filosofía en Colombia a partir de 1990}

Los principales cambios en el campo de la enseñanza de la filosofía en Colombia se dieron a partir de la década del 90. La Ley General de Educación (Ley 115 de 1994) precisó los objetivos de formación para los nuevos ciudadanos colombianos, de cara a las metas definidas en la Constitución Política de 1991; especialmente por lo estipulado en el artículo 67, que exigía un ciudadano crítico, participativo, con capacidad de reclamar sus derechos y comprometerse en el cumplimiento de sus deberes. Retornando a la Ley General, la educación se definió en su Artículo 1 como un proceso de formación permanente, personal, cultural y social que se fundamenta en una concepción integral de la persona humana, de su dignidad, de sus derechos y deberes. Sin entrar en detalles en la organización de la prestación del servicio educativo en los niveles básico y medio, interesa para nuestra reflexión que la filosofía aparece en el Artículo 31 como un área fundamental para la formación de ciudadanos que en educación media opten por el énfasis académico.

Pese a lo anterior, en las pruebas censales para acceder a la educación superior aplicadas a los bachilleres, seguía apareciendo la prueba de filosofía como obligatoria; algo que la tornaba exigible en la práctica para todas las modalidades de bachillerato. El contenido del área de filosofía no estaba definido, se contaba con un referente anterior con la Ley Uribe de 1903, en la que se dividió el bachillerato en técnico y clásico, al ubicar en el segundo la formación humanística en filosofía y letras. En ella, la formación filosófica era vista como aquella concentrada en el tratamiento de la metafísica, ontología, epistemología y, en algunos casos, ética o moral. Sin embargo, el vacio en términos curriculares y metódicos en este campo era notorio, a ello se enfrenta la Resolución 2343 de 1996, en la que se ofrecen ocho indicadores de logros para la enseñanza de la filosofía en la educación media. La educación filosófica de un bachiller colombiano, a la luz de la resolución mencionada, cultivaba procesos cognitivos vinculados en algunos casos a unos contenidos, asi como a algunas metas formativas definidas. Cabe anotar que en dichos indicadores no existe una jerarquización de los procesos cognitivos bajo el amparo de alguna taxonomía o alguna teoria del aprendizaje. 
Si bien se ofrecian estos indicadores de logros para elárea, no habia unos lineamientos curriculares que orientaran a los profesores en la selección de contenidosy la elección de metódicas y estrategias de enseñanza. A pesar de ello, resulta necesario recordar que esta resolución precede la propuesta de la serie Lineamientos Curriculares que para 1998 había definido el Ministerio de Educación Nacional en diferentes áreas. Lo que se evidenciaba era una tendencia hacia el enfoque del Aprendizaje Significativo y el Aprendizaje Basado en Problemas como el norte para pensar la educación en Colombia y que, además, se da en el marco de las transformaciones en educación propuestas desde la administración del presidente César Gaviria en 1990, cuyo objetivo principal fue la adopción del enfoque de competencias en educación, que condujo a una reconceptualización en 1995 de las pruebas censales que aplicaba el Instituto Colombiano para la evaluación de la Educación (ICFES) en aquel momento, pero que se sintió con mayores efectos en los exámenes que se implementaron a partir del año 2000, en los que se evaluaba la competencia comunicativa, como propone Bustamante (2003), desde una perspectiva de la lengua.

Volviendo a los referentes legales sobre la enseñanza de la filosofía en la década del 90 en Colombia, se observa que no existe una orientación única para la enseñanza de la filosofía en el bachillerato. A pesar de ello, nuevamente la prueba de filosofía hacia parte del examen censal aplicado por el ICFES para este periodo, en el que se retomaban los temas, problemas o autores propios de la filosofía desde una perspectiva histórica; hecho que, a la larga, se convirtió en derrotero para su enseñanza en las aulas. La filosofía, como indicamos, era tratada como un cuerpo de contenidos de manera histórica, lineal y teórica; y los campos filosóficos que solian evaluarse en ese momento eran cosmología, metafísica, moral, antropología, estética, epistemología y lógica.

Para el año 2000, acontece uno de los principales cambios en la educación en Colombia: la adopción directa del enfoque de competencias para la planeación y el diseño curricular. A partir de ese momento, la meta era impulsar un sistema educativo que en los niveles de educación básica y media fortaleciera competencias comunicativas de carácter general en todas la áreas (interpretativa, argumentativa y propositiva), de manera que se asumía la competencia como un saber hacer en contexto. Para el caso de la filosofía, implicó un giro en la prueba ICFES, en la que los 
campos por evaluar se redujeron a los siguientes: ética, antropología, epistemología, ontología y estética. También en ciertas ocasiones se pedía a los estudiantes tomar postura frente a algunas teorias filosóficas. Si bien el panorama parecia alentador, muchas de las criticas a estas pruebas radicaban en la especialidad del lenguaje empleado, la incapacidad de poder evaluar contextos particulares de los estudiantes y la concentración en una filosofía vista desde una perspectiva histórico-lineal y centrada en el pensamiento occidental.

Durante seis años ese fue el norte que orientó la evaluación de la filosofía en la educación media en Colombia y, por defecto, su enseñanza. El año 2007 sería el que marcaría otro de sus grandes cambios, pues aparece un documento emitido por el ICFES, titulado Fundamentación conceptual área de Filosofía, de la autoría de Sofía Melo, bajo la supervisión de Carlos Augusto Hernández y Margarita Torrez. Este documento retoma directamente la definición de la UNESCO sobre competencias ofrecida en el año 2000, a partir de los siguientes cuatro pilares de la educación: Aprender a conocer, Aprender a hacer, Aprender a Ser y Aprender a vivir juntos. La filosofía desempeñaria un papel central en ello; por eso, según Melo (2007), se debía dar un salto de una educación filosófica centrada en lo ético, lo antropológico, lo epistemológico y lo estético a una propuesta en cuyo núcleo estuviesen preguntas transversales por el hombre, el ser y el conocimiento. Un hecho como este condujo a postular no solo una definición de filosofía, sino también a proponer una estructura para su enseñanza, unas preguntas para la planeación docente, unos parámetros de evaluación y, finalmente, una idea de formación filosófica.

Melo ofrece, desde nuestro punto de vista, seis matices que ayudan a comprender la definición de filosofía para la educación media en Colombia y que son capaces de responder a las demandas de la UNESCO. El primero de ellos es que la filosofía es vista como caminar hacia el conocimiento y develamiento de su ser, por medio de la interpretación de la existencia; pero, para lograrlo, es necesario rescatar su historia. El segundo que la filosofía permite que nuestro mundo, nuestros pensamientos y nuestras acciones tengan sentido o valor, lo que se traduce en la defensa de la formación de un individuo ético, responsable y libre. 
El tercero que la filosofía debe ocuparse de recuperar el contexto del estudiante y apoyarse en la base conceptual del área. En ese sentido, era menester poner en el centro de la formación los diálogos estudiante-contexto, estudiante-política, religión-arte y estudiante-existencia. El cuarto que la filosofía es vista como un espacio de reflexión, de crítica, argumentación y discusión de los estudiantes colombianos; por eso, resultaba necesario fortalecer la interpretación, al ser la que ayuda a reconstruir el mundo y contribuye a la constitución de la identidad.

El quinto que la filosofía es asumida como un saber que promueve una actitud que cuestiona y resulta crítica frente a la vida. Aquí se postula una imagen de hombre como un ser racional e integrado con el mundo. El sexto propone que el pensar filosófico se entienda como la capacidad de reflexionar criticamente en torno a las condiciones constitutivas de la realidad y su existencia. Para lograr lo anterior, es necesario fortalecer el diálogo con la historia y las condiciones de producción de las diferentes teorias.

Para organizar su enseñanza, era necesario, en primer lugar, partir de unos problemas filosóficos vinculados a ciertos autores y a unos contextos específicos que se combinaban con una perspectiva histórica general, en la que se pusiera en evidencia la trascendencia de dichos problemas y con la vida cotidiana de los alumnos. En segundo lugar, elegir unos recursos: los que se sugerian para los profesores de filosofía en la educación media eran los textos filosóficos, las matrices culturales, las experiencias cotidianas del profesor, los estudiantes y la comunidad. Finalmente, en tercer lugar, las competencias por evaluar serian las interpretativas, argumentativas y propositivas, pero delimitadas para el área.

Las preguntas para la planeación en el área de filosofía estaban centradas en tres asuntos: A. Las preguntas del hombre frente a su mundo social y cultural (Cultura, arte, pautas morales, lenguaje y teorias del poder). B. Las preguntas por el ser (Ser, mundo, hombre, hombre y totalidad). C. Las preguntas por el conocimiento (Ciencia, realidad y sociedad). La evaluación debía centrarse en la capacidad de los estudiantes para enfrentarse a los textos filosóficos, identificar su vínculo con las matrices culturales y emplear los conceptos filosóficos en el análisis de situaciones cotidianas. 
Los textos filosóficos siguen ocupando un lugar central, porque las lecturas son asumidas como herramientas conceptuales que le permiten al estudiante analizar y confrontar posiciones. Finalmente, Melo (2007) propone que la formación filosófica procure que el estudiante colombiano, con base en el patrimonio filosófico de la humanidad, genere herramientas conceptuales, por medio de las cuales piense las diferentes dimensiones de la realidad y de su existencia personal y social como sujeto, con derechos y deberes, perteneciente a un contexto.

Durante cuatro años estuvo en marcha esta nueva reconceptualización para el área de filosofía y para la prueba de filosofía que hacia parte del examen ICFES. Nuevamente, no existía una orientación general para los docentes. Con el fin de enfrentar esta situación, fruto de un trabajo colaborativo entre universidades y docentes de filosofía de diferentes regiones del país, el Ministerio de Educación Nacional (MEN) emite el Documento No. 14: Orientaciones Pedagógicas para la Filosofía de la Educación Media (Gaitán y otros, 2010). Alli se plantea que su finalidad es la formación de sujetos dialógicos, críticos y hermeneutas de su época, algo que se logra a través de estrategias grupales e individuales: la comunidad de indagación y la disertación, por ejemplo. También se fijaron áreas para el tratamiento de este saber escolar. A partir de 2010, se recomienda que los profesores de filosofía se concentren en los campos estético, epistemológico y político.

El desarrollo, fortalecimiento y consolidación de unas competencias básicas (comunicativas, matemáticas, científicas y ciudadanas) se pretende poner en marcha a partir de los conocimientos y habilidades propios del campo filosófico y de las habilidades que éste requiere, a saber: la indagación y reflexión en torno a un fenómeno o problema de la naturaleza, la sociedad o del pensamiento en general. Lo que se persigue es que el estudiante proponga soluciones de manera argumentada. En su orden, las competencias filosóficas son la crítica, la creativa y la dialógica. Pero esta propuesta está acompañada de estrategias didácticas para plantear problemas filosóficos desde la moral, la epistemología y la estética en los contextos propios de los docentes y de los estudiantes. La implementación de dichas estrategias es la que dará cabida a la reflexión y a la construcción del sentido de la filosofía en calidad de disciplina que se centra en los problemas propios de la condición humana o de nuestras acciones, nuestra relación con el conocimiento y el cultivo de la sensibilidad artística. 
En sintesis, a la filosofia le corresponde la tarea de promover el pensamiento crítico y el creativo, de tal modo que sea posible una reflexión autónoma, en paralelo con el cultivo de la comunicación como forma esencial de interacción social. Si se logra desarrollar en el estudiante las competencias dialógica, crítica y creativa, podrá esperarse un sujeto educado capaz de pensar y experimentar asuntos como la certeza, la justicia y la belleza; y, de igual manera, construir nexos con "situaciones reales de nuestra sociedad, tales como la violencia, las formas de dogmatismo religioso, ideológico y político; la violación de los derechos humanos, la intolerancia ética y política; la indiferencia frente a las exigencias que plantea la formación ciudadana" (Gaitán, López, Quintero y Salazar, 2010, p. 24).

Así las cosas, en 2010, parece que el MEN considera fundamental la enseñanza de la filosofía, porque a través de ella es posible aprender a filosofar. La meta es cultivar unas cualidades y competencias que permitan reflexionar sobre los problemas propios, tanto individual como colectivamente. De ahora en adelante, ya no bastará con reconocer unos hitos históricos en el pensamiento filosófico, algunas frases o autores, sino que se tratará de impulsar en la clase de filosofia el uso de argumentos claros y la puesta en marcha de un diálogo respetuoso que responda a las necesidades formativas del estudiante de educación media en Colombia. Así, la historia de la tradición filosófica y el ejercicio mismo del filosofar se conjugan de la mejor manera.

En últimas, se dice en el texto que:

La enseñanza de la filosofía sirve para formar sujetos autónomos, amantes del diálogo y de la creatividad, lo cual enriquece sin duda el desarrollo de las competencias básicas; y como éstas son indispensables para vivir en el mundo contemporáneo, entonces el aumento de su desarrollo, procurado por el de las especificas de la filosofía, resulta sumamente útil (Gaitán et al., 2010, p. 38).

Esto será retomado en la prueba SABER 11, en la que los estudiantes debian responder en filosofía a preguntas sobre el conocimiento, la estética y la moral, bajo el enfoque de competencias específicas y en situaciones de su cotidianidad. 
Nuevamente, hay cambios orientados desde elICFESyen 2013 se emite eldocumento Alineación del examen SABER 11. Desde ese año, lo que se evalúa a los estudiantes colombianos son competencias genéricas que incluyen referentes de todas las disciplinas. Estas se entienden como aquellas que se desarrollan a lo largo del proceso educativo y que "resultan indispensables para el desempeño social, laboral y cívico de todo ciudadano, independientemente de su oficio o profesión" (ICFES, 2013, p. 12.) Las competencias genéricas han sido catalogadas como "competencias para la vida" que preceden, atraviesan y se complementan con las competencias no-genéricas propias de oficios o actividades laborales particulares, producto de un trabajo especializado constante. Sin las competencias genéricas, no podriamos contar con alguien que tenga éxito en lo laboral y con capacidad de participar en la vida social y política de la comunidad a la que pertenece. Como mencionamos, algo también en lo que insiste el ICFES, son competencias que se desarrollan a lo largo del proceso educativo y que para el contexto colombiano han sido aceptadas bajo la denominación de competencias en lectura, escritura, matemáticas y ciudadanía.

En el caso del área de filosofia, al hacer una revisión de las pruebas anteriores y de las reflexiones sobre las competencias vinculadas a la filosofía, propone el ICFES que para poder ajustarse a la evaluación de competencias genéricas resulta necesario fusionarla con el área de lenguajes en la nueva prueba para grado once denominada SABER 11, toda vez que ambas áreas buscan evaluar el nivel de lectura de los estudiantes y solo se diferencian por el tipo de textos que utiliza cada prueba. En cuanto a asuntos de forma, el ICFES decide incluir más preguntas por prueba, así como preguntas abiertas en el examen SABER 11 a partir de 2013; a lo que se agrega la eliminación del componente flexible del examen si se diseña propiamente como una prueba estandarizada.

En sintesis, la prueba de lectura crítica evalúa las capacidades que tiene un estudiante colombiano para entender, interpretar y evaluar información. La orientación para las áreas de Filosofía y Lenguaje en educación básica y media es que formen estudiantes capaces de reconocer información en los textos, contextualizarla y usarla para pensar problemas cotidianos. Los textos que se emplean para la prueba son textos de la vida cotidiana y académicos no especializados, que pueden ser de carácter continuo y que exigen una habilidad para reconstruir la línea argumentativa 
del texto (frases, párrafos, capítulos). También pueden ser discontinuos, pues exigen una habilidad para asociar información y extrapolar el análisis (cuadros, imágenes, gráficos, tablas, fragmentos de textos, etc.). Además, están los textos literarios e informativos. Con todo esto se lograría evaluar el nivel del estudiante en la competencia de Comprensión lectora, entendida como aquella que le permite interpretar, aprender y tomar posturas criticas frente a un texto.

Muchas tensiones genera el giro de las pruebas SABER 11, en las que el área de Filosofía desaparece como prueba especíica. Se abre el campo hacia un trabajo transversal con el área de Lengua Castellana, que exige una rearticulación del sentido que tiene el saber filosófico en la formación de los estudiantes que llegan a grado 11. Las discusiones están a la orden del día, no necesariamente en lo que toca a la desaparición del área de Filosofía, sino a la transformación que acontecerá dentro de su enseñanza, si consideramos que se pide que ayude a los estudiantes a evaluar su comprensión de los textos y el contenido de estos en una estrecha conexión con su contexto. ¿Qué tal si pensamos en una formación filosófica que permita el cultivo de una competencia en Lectura Crítica, desde una perspectiva latinoamericana, como eje para el diseño de experiencias educativas en la educación media colombiana? Precisamente de eso nos ocuparemos en el siguiente apartado.

\section{Por una alternativa para la enseñanza de la filosofía en Colombia}

Atendiendo a la necesidad de pensar una alternativa para la enseñanza-aprendizaje de la filosofía en Colombia, se proponen las Orientaciones Pedagógicas para la Filosofía desde Nuestramérica (OPFN), las cuales son el resultado del trabajo de investigación al interior de la Maestria en Educación ofrecida por la Universidad de Antioquia (Seccional Oriente) en su cohorte IV. Estas Orientaciones parten de un problema fundamental: el lugar común para la enseñanza-aprendizaje de la filosofía en la escuela suele estar determinado por el pensamiento europeo impuesto desde la modernidad como la única manera legitima y universal de hacer filosofía y, por tanto, como la única filosofía digna de ser conservada y transmitida al interior de las diferentes culturas. 
Fundamentadas en una perspectiva liberadora, critica y decolonial, en las OPFN se argumenta que, debido a los procesos históricos de colonización, los ideales de la modernidad europea fueron impuestos como el camino para alcanzar la civilización y la humanidad al interior de las distintas culturas periféricas, lo que da lugar a la hegemonía del eurocentrismo presente en las actuales condiciones del sistema-mundo y la globalización, en las que las relaciones de dominación y dependencia aún prevalecen bajo la colonialidad del poder, del ser, del saber y de la naturaleza. Estas expresiones coloniales han conducido al silenciamiento de la diversidad de sabidurias y prácticas culturales presentes en los territorios de la periferia y, asimismo, de otras formas de pensamiento filosófico que dan cuenta de la particularidad histórica y social de los pueblos.

La escuela y los maestros de filosofía han contribuido a reforzar estas expresiones de la colonialidad cuando diseñan sus mallas curriculares y planes de estudio para la filosofía en la Educación Media, a partir de las pautas sugeridas por el MEN en su Documento No. 14: Orientaciones Pedagógicas para la Filosofía de la Educación Media (Gaitán et al., 2010), o con base en otra serie de libros de texto, en los que se trabaja desde estándares conceptuales y de competencias generales que refuerzan las pretensiones de una universalidad de la filosofía eurocéntrica, al atender a sus cuatro momentos históricos: clásica, medieval, moderna y contemporánea; o pensados a partir de problemas clasificados en las distintas disciplinas de la filosofía: cosmología, ontología, antropología, epistemología, ética, política, estética, entre otras. Lo anterior resulta válido, pero tiene como principal limitación que las referencias bibliográficas suelen ser los filósofos hombres de Europa o Norteamérica $y$, cuando de pronto se tienen en cuenta otras perspectivas o expresiones filosóficas surgidas alrededor del mundo, se hace como un apéndice o una nota al margen.

Por tanto, la invitación es a atrevernos a sentipensar desde nosotros mismos, a interpelar la hegemonía del eurocentrismo, a des-silenciar las distintas cosmovisiones y filosofías presentes en Nuestramérica, para dar lugar asi a un pensamiento filosófico, crítico, liberador y decolonial. Es por esta razón que las OPFN acuñan la categoría de Nuestramérica, al tener como referente la obra del filósofo cubano José Martí (1903), titulada Nuestra América, en la que se hace un llamado a tomar conciencia de nuestra condición social de dependencia y de la necesidad 
de emancipación cultural. Nuestramérica, por su parte, profundiza un poco más el sentido y le otorga actualidad, al hacer alusión a nuestro devenir histórico, las cosmovisiones, los pluriversos, los mundos relacionales, las luchas de resistencia contra la colonización y las condiciones de colonialidad y, en última instancia, la búsqueda de un pensamiento filosófico propio desde el cual generar un diálogo interfilosófico e intercultural.

Atendiendo a esta problemática, se planteó el siguiente objetivo general para el trabajo de investigación:

Analizar los fundamentos teóricos y conceptuales presentes en el pensamiento filosófico de Nuestramérica y en la pedagogía crítica y decolonial, con el fin de proponer algunas orientaciones pedagógicas para el área de filosofía en la Educación Media que contribuyan al análisis crítico en torno a los problemas y las competencias básicas planteadas por el MEN (Carmona-Cardona, 2018, p. 21).

Lo anterior permitió arrojar como resultado una alternativa curriculary didáctica para la enseñanza-aprendizaje de la filosofía, centrada en el empoderamiento por parte de los estudiantes de unas competencias básicas que apunten hacia una formación para el buen vivir de todos los seres humanos en armonía con la naturaleza. Para lograr esto, las OPFN se desarrollaron a partir de cuatro momentos fundamentales: Componente Filosófico, Componente Pedagógico, Aspectos Curriculares y Aspectos Didácticos.

El Componente Filosófico fue construido con base en las periodizaciones históricas elaboradas por Gaos (1944), Roig (2004), Dussel, (1994) y Beorlegui (2010). Las susodichas sirvieron como cartas de navegación para el análisis de la historicidad del pensamiento filosófico de Nuestramérica, así como de los principales filósofos, pensadores e intelectuales y también de los problemas y categorias más importantes que los han ocupado. Se parte asi de una postura afirmativa que defiende la existencia de un pensamiento filosófico auténtico más allá de la tradición filosófica europea, centrado en el análisis de la propia situación histórica, las circunstancias sociales y los problemas culturales, contrario a una postura asuntiva que defiende la universalidad de la filosofia como característica inherente al legado europeo. 
Este acercamiento de carácter historicista y afirmativo permitió identificar dos momentos fundamentales, nombrados como Tradición Filosófica de Nuestramérica y Paradigmas Filosóficos Contemporáneos de Nuestramérica, los cuales son ilustrados brevemente por medio de la siguiente tabla:

Geo-historicidad del pensamiento filosófico de Nuestramérica

Tradición Filosófica de Nuestramérica

\begin{tabular}{|c|c|}
\hline Periodos & Algunos Autores \\
\hline \multicolumn{2}{|c|}{ Colonización y dominación (s. XV-XVIII) } \\
\hline 1492-1553: Política colonial & Bartolomé de Las Casas \\
\hline $\begin{array}{l}\text { 1553-1750: Primera normalización } \\
\text { filosófica }\end{array}$ & $\begin{array}{l}\text { Bartolomé de Las Casas, Alonso de la } \\
\text { Vera Cruz }\end{array}$ \\
\hline $\begin{array}{l}\text { 1750-1807: Crisis de la ilustración y } \\
\text { transición a la modernidad }\end{array}$ & Benito Díaz, Manuel Paramás \\
\hline \multicolumn{2}{|c|}{ En búsqueda de la emancipación (s. XIX) } \\
\hline 1807-1820: Emancipación cultural & Simón Bolivar, Juan Alberdi \\
\hline $\begin{array}{l}\text { 1820-1870: Influencia Franco- } \\
\text { Anglosajona, transición liberal y } \\
\text { romanticismo ilustrado }\end{array}$ & Andrés Bello, Domingo Sarmiento \\
\hline $\begin{array}{l}\text { 1870-1900/1910: Krausismo y } \\
\text { Positivismo }\end{array}$ & Eugenio Hostos, José Ingenieros \\
\hline $\begin{array}{l}\text { 1870-1900/1910: Emancipación } \\
\text { auténtica }\end{array}$ & José Martí \\
\hline \multicolumn{2}{|c|}{ La segunda normalización filosófica (s. XX) } \\
\hline $\begin{array}{l}\text { Generación de } 1900 \text { y 1915: Patriarcas o } \\
\text { fundadores }\end{array}$ & $\begin{array}{l}\text { José Rodó, José Mariátegui, José } \\
\text { Vasconcelos }\end{array}$ \\
\hline $\begin{array}{l}\text { Generación de 1927-1939: Exiliados } \\
\text { españoles, Forjadores o Normalización }\end{array}$ & Samuel Ramos, José Gaos \\
\hline $\begin{array}{l}\text { Generación de 1945: Discípulos de los } \\
\text { forjadores }\end{array}$ & Leopoldo Zea, Augusto Salazar \\
\hline $\begin{array}{l}\text { Generación de 1969: Filosofía de la } \\
\text { liberación }\end{array}$ & Enrique Dussel, Leonardo Boff \\
\hline
\end{tabular}




\begin{tabular}{|l|l|l|}
\hline \multicolumn{4}{|c|}{ Paradigmas Filosóficos Contemporáneos de Nuestramérica } \\
\hline \multicolumn{1}{|c|}{ Corrientes } & \multicolumn{1}{|c|}{ Breve Descripción } & Algunos Autores \\
\hline $\begin{array}{l}\text { Filosofia de la } \\
\text { liberación }\end{array}$ & $\begin{array}{l}\text { Búsqueda de caminos para el } \\
\text { surgimiento de organizaciones civiles y } \\
\text { democráticas que luchen por el respeto, } \\
\text { el des-silenciamiento y la inclusión. }\end{array}$ & $\begin{array}{l}\text { Enrique Dussel, } \\
\text { Horacio Cerutti }\end{array}$ \\
\hline $\begin{array}{l}\text { Filosofía } \\
\text { intercultural }\end{array}$ & $\begin{array}{l}\text { Propende por una superación del logos } \\
\text { hegemónico de la filosofía europea } \\
\text { como condición para generar una } \\
\text { apertura dialogal auténtica con lo otro. }\end{array}$ & $\begin{array}{l}\text { Raúl Fornet- } \\
\text { Betancourt, David } \\
\text { Mora }\end{array}$ \\
\hline $\begin{array}{l}\text { Pensamiento } \\
\text { decolonial }\end{array}$ & $\begin{array}{l}\text { Pensamiento critico que denuncia la la } \\
\text { colonialidad del poder, del ser, del saber } \\
\text { y de la naturaleza como resultado de la } \\
\text { modernidad, proponiendo en su lugar } \\
\text { la diversidad epistémica. }\end{array}$ & $\begin{array}{l}\text { Grupo } \\
\text { Modernidad/ } \\
\text { Colonialidad: } \\
\text { Anibal Quijano, } \\
\text { Walter Mignolo, } \\
\text { Edgardo Lander, } \\
\text { Raúl Grosfoguel, } \\
\text { Arturo Escobar, } \\
\text { Catherine Walsh }\end{array}$ \\
\hline $\begin{array}{l}\text { Posmodernidad en } \\
\text { Nuestramérica }\end{array}$ & $\begin{array}{l}\text { «Posmodernidad» es un cambio de } \\
\text { sensibilidad al nivel del mundo de } \\
\text { la vida que se produce no sólo en las } \\
\text { regiones centrales de Occidente, sino } \\
\text { también en las periféricas. }\end{array}$ & $\begin{array}{l}\text { Santiago Castro- } \\
\text { Gómez }\end{array}$ \\
\hline
\end{tabular}

-Nota: Elaboración propia, propuesta a modo de sintesis del Componente Filosófico de las OPFN, adaptada a partir de la «Tabla 5: Geo-historicidad del pensamiento filosófico de Nuestramérica» (Carmona-Cardona, 2018, pp. 143-146)

Este marco geo-históricoy geo-referencial del pensamiento filosófico de Nuestramérica, sumado al necesario des-silenciamiento de las cosmovisiones presentes en las culturas precolombinas: Náhuatl, Maya, Muisca, Inca se presenta como el paradigma necesario para establecer un diálogo interfilosófico e intercultural con las demás filosofías del mundo, lo que da lugar a una nueva etapa histórica de la filosofía, en términos de lo que Dussel (2009) nombra como un pluriverso transmoderno. 
Ahora bien, como el problema que acá compete es el de la enseñanza-aprendizaje de la filosofía en la educación media, también se hizo necesario desarrollar un Componente Pedagógico desde una perspectiva crítica y decolonial, pues por las particularidades históricas y sociales del pensamiento filosófico de Nuestramérica una pedagogía pensada en términos tradicionales y eurocéntricos no estaría acorde con lo planteado. Por eso se apuesta por la teoría crítica latinoamericana, dado que en ella pueden encontrarse los fundamentos pedagógicos para justificar la necesidad de una filosofía de Nuestramérica en la escuela.

En torno a la importancia de valorar la educación desde una perspectiva crítica, señala Mejía (2011) lo siguiente:

Parte de la idea de interculturalidad, reconoce la existencia de otros saberes existentes en las culturas colonizadas de África, Asia y América Latina, las cuales son negadas por la existencia de un conocimiento hegemónico de tipo eurocéntrico, el cual controla a partir de la colonialidad que se da en cuerpos, conocimientos, deseos, haciéndonos como euro-estadounidenses. Por ello, proclama la búsqueda de una educación propia que permita un diálogo de saberes, que visibiliza esas otras racionalidades y cosmovisiones, lo cual permite cuestionar la forma universal en que se mueve el conocimiento euro-estadounidense (Citado por CarmonaCardona, 2018, p. 75).

La educación pensada desde una perspectiva crítica y decolonial permite confrontar las premisas de una educación neoliberal, al proponer la búsqueda esperanzadora de la plenitud del ser humano y del buen vivir para todos. Las condiciones sociales del mundo no están determinadas por necesidad; por el contrario, son contingentes, de ahí que el ser humano pueda intervenirlo y transformarlo. Esta esperanza de transformación se fundamenta en el principio de que "cambiar es difícil pero posible" (Freire, 2012, p. 119). La educación se convierte asi en una herramienta para el cambio y, por tal razón, está articulada con la política, dado que no es neutral. De ahí la necesidad de que los maestros y maestras de filosofía sean conscientes de la dimensión política de la educación y se comprometan con la promoción de un diálogo equitativo entre los distintos saberes y filosofías, así como con la formación de ciudadanías libres, la defensa de la democracia y la lucha por un mundo más humano y justo. 
A partir de los componentes filosófico y pedagógico, se obtuvo la fundamentación teórica necesaria para proponer algunos aspectos curriculares y didácticos para la enseñanza- aprendizaje de la filosofía en la educación media, con base en el pensamiento filosófico de Nuestramérica y desde una pedagogia critica y decolonial.

Los Aspectos Curriculares fueron propuestos en perspectiva crítica e intercultural principalmente, en diálogo con los trabajos de Apple (2008), Gimeno (2008) y Torres (1998) con el fin de cuestionar la hegemonia de los saberes eurocéntricos que predominan en los currículos escolares, e invitando, al mismo tiempo, al dessilenciamiento de un pensamiento filosófico alternativo necesario para el diálogo interfilosófico e intercultural. Las reflexiones curriculares están orientadas por tres preguntas que le son inherentes: “¿Para qué enseñar? ¿Qué enseñar? ¿Desde dónde enseñar?" (Carmona-Cardona, 2018, p. 110).

La pregunta ¿para qué enseñar? conlleva a reflexionar en torno a los objetivos de la enseñanza-aprendizaje de la filosofía, que para el caso colombiano están planteados en términos de "competencias". Esta categoria suele ser bastante compleja, en razón de sus múltiples significados. Si bien es cierto que suele ser empleada en los ámbitos laborales y que su adaptación al ámbito educativo se hizo con el fin de establecer estándares de calidad que dieron lugar a un enfoque utilitario de las competencias al servicio de la educación neoliberal también es cierto que, de acuerdo con Gimeno (2008), es posible pensar dicha categoría desde la perspectiva del desarrollo humano, lo cual permite reinterpretarla como un concepto holístico que no se agota en el desarrollo de habilidades para el trabajo, sino que propende además por una educación integral que permita el desarrollo de conocimientos, destrezas y actitudes susceptibles de ser aplicados en diferentes contextos y situaciones más allá del espacio escolar.

Fue así como se llegó al planteamiento de cuatro competencias básicas, en diálogo crítico con las competencias propuestas por el MEN y el ICFES. Dichas competencias básicas son las siguientes: 
- Conciencia critica (Saber): Articulada con la competencia interpretativa del ICFES y la competencia crítica del MEN. Busca concientizar a los estudiantes respecto a las actuales condiciones de dominación y colonialidad, presentes tanto en las estructuras de conocimiento hegemónicas como en los sistemas económicos y políticos de corte neoliberal.

- Diálogo interfilosófico (Saber-Hacer): Articulada con la competencia argumentativa del ICFES y la competencia dialógica del MEN. Su finalidad es interpelar la homogeneización cultural, con el fin de des-silenciar las distintas filosofias, saberes y sentipensamientos de Nuestramérica, al convocar a una ecología de saberes en condiciones horizontales.

- $\quad$ Acción transformadora (Saber-Ser): Articulada con la competencia propositiva del ICFES y la competencia creativa del MEN. Parte del principio de que el ser humano se encuentra en un estado de continua interrelación con el mundo, en el que el conocimiento y las estructuras sociales son susceptibles de ser recreadas a partir de las necesidades del contexto.

- $\quad$ Ciudadanía intercultural (Saber-Convivir): Articulada con las competencias ciudadanas del MEN. Propende por una formación en valores ciudadanos de carácter intercultural, necesarios para el ejercicio de la democracia bajo las actuales dinámicas de mundialización, caracterizadas por los encuentros e intercambios entre diferentes culturas.

Otro elemento fundamental de los Aspectos Curriculares es propuesto a partir de las preguntas ¿qué enseñar? y ¿desde dónde enseñar? La respuesta es clara: Filosofía desde Nuestramérica. Pero el problema que acá compete es la posibilidad de sugerir algunos ejes problemáticos y categorias de análisis que permitan llevar a cabo la enseñanza-aprendizaje de la filosofía desde esta perspectiva. En este orden de ideas, se propone lo siguiente: 
Matriz categorial: Sentipensando filosóficamente desde Nuestramérica

\begin{tabular}{lll}
\hline $\begin{array}{l}\text { Unidades } \\
\text { hermenéuticas }\end{array}$ & Ejes problemáticos & Categorias \\
\hline 1. Lo histórico & Geo-historicidad del & Filosofía mundial \\
& pensamiento filosófico & Pueblos originarios \\
& & Filosofía colonial \\
& & Búsqueda de emancipación \\
& Normalización filosófica \\
& & Nuevos paradigmas \\
\hline epistemológico Lo & Decolonialidad del ser \\
& Epistemologías del Sur & Positivismo científico \\
& & Eurocentrismo \\
& & Decolonialidad del saber \\
& & Decolonialidad de la naturaleza \\
& & Cosmovisiones \\
& & Epistemologías otras \\
& & Diálogo interfilosófico- \\
intercultural
\end{tabular}

Nota: Elaboración propia (Carmona-Cardona, 2018, p. 140). 
Finalmente, en las OPFN, se desarrollan unos Aspectos Didácticos a partir de la pregunta en torno a ¿Cómo enseñar? En función de ésta, se plantea una propuesta didáctica crítica para la filosofía desde Nuestramérica, centrada en la necesidad de dar lugar a la reflexión filosófica al interior del aula de clase y con base en preguntas problematizadoras en torno a los ejes problemáticos y las categorias sugeridas en los Aspectos Curriculares. Asimismo, se proponen una serie de estrategias didácticas para la enseñanza-aprendizaje de la filosofía, con el fin de que los estudiantes puedan desarrollar las competencias filosóficas básicas a partir de ejercicios de lectura, escritura, oralidad, indagación y el uso de herramientas tecnológicas.

Los Aspectos Didácticos están conformados también por el Esquema General de la Propuesta Curricular y por la Malla Curricular, en los que se articulan las unidades hermenéuticas, los ejes problemáticos, los ámbitos conceptuales, las preguntas problematizadoras, las competencias y los indicadores de desempeño. La siguiente tabla presenta una síntesis de la propuesta curricular para la filosofía desde Nuestramérica en la educación media.

Esquema general de la propuesta curricular:

\begin{tabular}{|l|l|l|l|}
\hline \multicolumn{3}{|l|}{$\begin{array}{l}\text { Grado 10: Núcleo de problema filosófico 1 } \\
\text { Lo histórico: Geo-historicidad del pensamiento filosófico }\end{array}$} \\
\hline $\begin{array}{l}\text { Competencia } \\
\text { filosófica }\end{array}$ & Desempeños & $\begin{array}{l}\text { Preguntas } \\
\text { filosóficas }\end{array}$ & $\begin{array}{l}\text { Ámbitos conceptuales } \\
\text { y contenidos generales }\end{array}$ \\
\hline $\begin{array}{l}\text { Conciencia } \\
\text { critica }\end{array}$ & $\begin{array}{l}\text { 1. Examina las } \\
\text { principales } \\
\text { caracteristicas } \\
\text { del pensamiento } \\
\text { filosófico, } \\
\text { identificando las } \\
\text { formas como éste ha } \\
\text { cobrado vida en los } \\
\text { diferentes contextos } \\
\text { históricos y culturales. }\end{array}$ & $\begin{array}{l}\text { 1. ¿Existen } \\
\text { formas otras de } \\
\text { pensamiento } \\
\text { filosófico } \\
\text { más allá de } \\
\text { la tradición } \\
\text { europea? }\end{array}$ & $\begin{array}{l}\text { 1. Filosofias del mundo } \\
\text { 1.1. Pensamiento } \\
\text { filosófico africano } \\
\text { 1.2. Pensamiento } \\
\text { filosófico asiático }\end{array}$ \\
\hline
\end{tabular}




\begin{tabular}{|c|c|c|c|}
\hline $\begin{array}{l}\text { Diálogo } \\
\text { interfilosófico }\end{array}$ & $\begin{array}{l}\text { 2. Evalúa los } \\
\text { principales } \\
\text { postulados del } \\
\text { pensamiento } \\
\text { filosófico } \\
\text { eurocéntrico, } \\
\text { estimando la } \\
\text { necesidad de un } \\
\text { diálogo interfilosófico } \\
\text { e intercultural en } \\
\text { el orden de una } \\
\text { ecología de saberes. }\end{array}$ & $\begin{array}{l}\text { 2. ¿Por qué se } \\
\text { plantea desde } \\
\text { una perspectiva } \\
\text { decolonial } \\
\text { la necesidad } \\
\text { de superar } \\
\text { los limites } \\
\text { impuestos por } \\
\text { el pensamiento } \\
\text { filosófico } \\
\text { eurocéntrico? }\end{array}$ & $\begin{array}{l}\text { 2. Filosofía europea } \\
\text { 2.1. Filosofía clásica y } \\
\text { medieval } \\
\text { 2.2. Filosofía moderna y } \\
\text { contemporánea }\end{array}$ \\
\hline $\begin{array}{l}\text { Acción } \\
\text { transformadora }\end{array}$ & $\begin{array}{l}\text { 3. Propone } \\
\text { alternativas de } \\
\text { sentipensamiento } \\
\text { filosófico que } \\
\text { permitan des- } \\
\text { silenciar formas de } \\
\text { saber otras, acordes } \\
\text { con el contexto } \\
\text { histórico y las } \\
\text { necesidades socio- } \\
\text { culturales. }\end{array}$ & $\begin{array}{l}\text { 3. ¿Hasta } \\
\text { qué punto el } \\
\text { pensamiento } \\
\text { filosófico está } \\
\text { determinado } \\
\text { por su contexto } \\
\text { histórico y socio- } \\
\text { cultural? }\end{array}$ & $\begin{array}{l}\text { 3. Nuestramérica: De } \\
\text { los pueblos originarios } \\
\text { hasta la independencia } \\
\text { de las Repúblicas } \\
\text { 3.1. Cosmovisiones de } \\
\text { los pueblos originarios } \\
\text { 3.2. Filosofía colonial y } \\
\text { emancipación }\end{array}$ \\
\hline $\begin{array}{l}\text { Ciudadanía } \\
\text { intercultural }\end{array}$ & $\begin{array}{l}\text { 4. Participa de manera } \\
\text { activa en el fomento } \\
\text { de los valores propios } \\
\text { de una ciudadania } \\
\text { intercultural, teniendo } \\
\text { como base la filosofía } \\
\text { de Nuestramérica y la } \\
\text { apuesta por un buen } \\
\text { vivir para todos los } \\
\text { seres humanos. }\end{array}$ & $\begin{array}{l}\text { 4. ¿Existe un } \\
\text { pensamiento } \\
\text { filosófico de } \\
\text { Nuestramérica } \\
\text { original y } \\
\text { auténtico, } \\
\text { a partir del } \\
\text { cual se pueda } \\
\text { establecer } \\
\text { un diálogo } \\
\text { interfilosófico? }\end{array}$ & $\begin{array}{l}\text { 4. Nuestramérica: De la } \\
\text { segunda normalización } \\
\text { filosófica hasta la } \\
\text { actualidad } \\
\text { 4.1. Siglo XX: La } \\
\text { búsqueda de una } \\
\text { filosofía original y } \\
\text { auténtica } \\
\text { 4.2. Nuevos paradigmas } \\
\text { filosóficos }\end{array}$ \\
\hline
\end{tabular}




\begin{tabular}{|c|c|c|c|}
\hline \multicolumn{4}{|c|}{$\begin{array}{l}\text { Grado 10: Núcleo de problema filosófico } 2 \\
\text { Lo epistemológico: Epistemologías del Sur para un diálogo interfilosófico e intercultural }\end{array}$} \\
\hline $\begin{array}{l}\text { Competencia } \\
\text { filosófica }\end{array}$ & Desempeños & \begin{tabular}{|l|} 
Preguntas \\
filosóficas \\
\end{tabular} & \begin{tabular}{|l} 
Ámbitos conceptuales \\
y contenidos generales
\end{tabular} \\
\hline $\begin{array}{l}\text { Conciencia } \\
\text { critica }\end{array}$ & $\begin{array}{l}\text { 1. Pone a prueba } \\
\text { la influencia } \\
\text { que ha tenido la } \\
\text { racionalidad técnica } \\
\text { en el desarrollo de las } \\
\text { sociedades y su nivel } \\
\text { de contribución para } \\
\text { un buen vivir. }\end{array}$ & $\begin{array}{l}\text { 1. ¿Ha } \\
\text { contribuido } \\
\text { verdaderamente } \\
\text { la racionalidad } \\
\text { técnica y el } \\
\text { conocimiento } \\
\text { científico al } \\
\text { bienestar de la } \\
\text { humanidad? }\end{array}$ & $\begin{array}{l}\text { 1. Teoria del } \\
\text { conocimiento } \\
\text { 1.1. El paradigma } \\
\text { científico del } \\
\text { conocimiento } \\
\text { 1.2. El positivismo en } \\
\text { Nuestramérica }\end{array}$ \\
\hline $\begin{array}{l}\text { Diálogo } \\
\text { interfilosófico }\end{array}$ & $\begin{array}{l}\text { 2. Debate en } \\
\text { torno a la manera } \\
\text { como, a partir del } \\
\text { paradigma científico } \\
\text { del conocimiento, } \\
\text { se ha dado lugar a } \\
\text { una colonialidad del } \\
\text { saber, promoviendo } \\
\text { al mismo tiempo } \\
\text { estrategias que } \\
\text { permitan alcanzar una } \\
\text { ecología de saberes. }\end{array}$ & $\begin{array}{l}\text { 2. ¿Es posible } \\
\text { hablar de } \\
\text { formas otras de } \\
\text { conocimiento } \\
\text { más allá del } \\
\text { paradigma } \\
\text { científico? }\end{array}$ & $\begin{array}{l}\text { 2. Pensamiento } \\
\text { decolonial } \\
\text { 2.1. Decolonialidad del } \\
\text { saber } \\
\text { 2.2. Decolonialidad de } \\
\text { la naturaleza }\end{array}$ \\
\hline $\begin{array}{l}\text { Acción } \\
\text { transformadora }\end{array}$ & $\begin{array}{l}\text { 3. Formula preguntas } \\
\text { problematizadoras } \\
\text { y caminos de } \\
\text { indagación que } \\
\text { conduzcan a } \\
\text { sentipensar } \\
\text { filosóficamente desde } \\
\text { Nuestramérica. }\end{array}$ & $\begin{array}{l}\text { 3. ¿Qué significa } \\
\text { y por qué es } \\
\text { importante } \\
\text { aprender a } \\
\text { sentipensar } \\
\text { desde nosotros } \\
\text { mismos? }\end{array}$ & $\begin{array}{l}\text { 3. Epistemologías otras } \\
\text { 3.1. Cosmovisiones y } \\
\text { otros mundos posibles } \\
\text { 3.2. La ecología de } \\
\text { saberes }\end{array}$ \\
\hline
\end{tabular}




\begin{tabular}{|c|c|c|c|}
\hline $\begin{array}{l}\text { Ciudadanía } \\
\text { intercultural }\end{array}$ & $\begin{array}{l}\text { 4. Promueve } \\
\text { el ejercicio de } \\
\text { una ciudadanía } \\
\text { intercultural a partir } \\
\text { de la valoración de } \\
\text { formas de pensar } \\
\text { otras presentes en } \\
\text { las cosmovisiones } \\
\text { andinas y los saberes } \\
\text { populares de } \\
\text { Nuestramérica. }\end{array}$ & $\begin{array}{l}\text { 4. ¿En qué radica } \\
\text { el valor de las } \\
\text { cosmovisiones } \\
\text { andinas y } \\
\text { los saberes } \\
\text { populares? }\end{array}$ & $\begin{array}{l}\text { 4. Diálogo } \\
\text { interfilosófico e } \\
\text { intercultural } \\
\text { 4.1. Por un pluriverso } \\
\text { transmoderno } \\
\text { 4.2. Interculturalidad } \\
\text { critica }\end{array}$ \\
\hline \multicolumn{4}{|c|}{$\begin{array}{l}\text { Grado 11: Núcleo de problema filosófico } 3 \\
\text { Lo práctico: Filosofia práctica para la liberación }\end{array}$} \\
\hline $\begin{array}{l}\text { Competencia } \\
\text { filosófica }\end{array}$ & Desempeños & $\begin{array}{l}\text { Preguntas } \\
\text { filosóficas }\end{array}$ & $\begin{array}{l}\text { Ámbitos conceptuales } \\
\text { y contenidos generales }\end{array}$ \\
\hline $\begin{array}{l}\text { Conciencia } \\
\text { crítica }\end{array}$ & $\begin{array}{l}\text { 1. Asume los } \\
\text { problemas de la } \\
\text { realidad social desde } \\
\text { una perspectiva } \\
\text { critica, identificando } \\
\text { las diferentes } \\
\text { relaciones de poder } \\
\text { existentes en el } \\
\text { marco del sistema- } \\
\text { mundo actual. }\end{array}$ & $\begin{array}{l}\text { 1. ¿Han } \\
\text { alcanzado en } \\
\text { realidad las } \\
\text { naciones de } \\
\text { Nuestramérica } \\
\text { una } \\
\text { independencia } \\
\text { en relación con } \\
\text { los países del } \\
\text { Norte? }\end{array}$ & $\begin{array}{l}\text { 1. Historicidad de las } \\
\text { ideas éticas y políticas } \\
\text { de Nuestramérica } \\
\text { 1.1. Colonialismo y } \\
\text { emancipación } \\
\text { 1.2. Normalización } \\
\text { Filosófica }\end{array}$ \\
\hline $\begin{array}{l}\text { Diálogo } \\
\text { interfilosófico }\end{array}$ & $\begin{array}{l}\text { 2. Juzga las actuales } \\
\text { politicas de una } \\
\text { economia y una } \\
\text { cultura globalizada, } \\
\text { propiciando } \\
\text { condiciones que } \\
\text { promuevan la } \\
\text { emancipación y el } \\
\text { diálogo intercultural } \\
\text { entre los pueblos. }\end{array}$ & $\begin{array}{l}\text { 2. ¿Las actuales } \\
\text { condiciones del } \\
\text { sistema-mundo } \\
\text { favorecen la } \\
\text { emancipación } \\
\text { de los pueblos o, } \\
\text { por el contrario, } \\
\text { contribuyen a } \\
\text { nuevas formas } \\
\text { de colonialidad? }\end{array}$ & $\begin{array}{l}\text { 2. El nuevo orden } \\
\text { geopolítico mundial } \\
\text { 2.1. Sistema-mundo } \\
\text { 2.2. Globalización }\end{array}$ \\
\hline
\end{tabular}




\begin{tabular}{|c|c|c|c|}
\hline $\begin{array}{l}\text { Acción } \\
\text { transformadora }\end{array}$ & $\begin{array}{l}\text { 3. Visualiza } \\
\text { alternativas de } \\
\text { solución para hacer } \\
\text { frente a las diferentes } \\
\text { desventajas del } \\
\text { sistema-mundo } \\
\text { actual y el capitalismo } \\
\text { neoliberal. }\end{array}$ & $\begin{array}{l}\text { 3. ¿Cómo } \\
\text { se pueden } \\
\text { superar las } \\
\text { condiciones de } \\
\text { alienación que } \\
\text { prevalecen aún } \\
\text { en los Estados } \\
\text { democráticos? }\end{array}$ & $\begin{array}{l}\text { 3. Ética y política para } \\
\text { la liberación } \\
\text { 3.1. Condiciones de } \\
\text { alienación } \\
\text { 3.2. Ética de la alteridad }\end{array}$ \\
\hline $\begin{array}{l}\text { Ciudadanía } \\
\text { intercultural }\end{array}$ & $\begin{array}{l}\text { 4. Colabora en } \\
\text { la construcción } \\
\text { de espacios } \\
\text { democráticos que } \\
\text { garanticen un buen } \\
\text { vivir para todos, } \\
\text { en medio de las } \\
\text { condiciones de una } \\
\text { sociedad globalizada. }\end{array}$ & $\begin{array}{l}\text { 4. ¿De qué } \\
\text { manera } \\
\text { contribuye el } \\
\text { pensamiento } \\
\text { decolonial a la } \\
\text { búsqueda de un } \\
\text { buen vivir? }\end{array}$ & $\begin{array}{l}\text { 4. Colonialidad/ } \\
\text { Decolonialidad del } \\
\text { poder } \\
\text { 4.1. La modernidad } \\
\text { colonial } \\
\text { 4.2. Imaginarios } \\
\text { coloniales del moderno } \\
\text { Estado-nación }\end{array}$ \\
\hline \multicolumn{4}{|c|}{$\begin{array}{l}\text { Grado 11: Núcleo de problema filosófico } 4 \\
\text { Lo estético: Estética del arte y de la vida cotidiana para una re-creación cultural }\end{array}$} \\
\hline $\begin{array}{l}\text { Competencia } \\
\text { filosófica }\end{array}$ & Desempeños & $\begin{array}{l}\text { Preguntas } \\
\text { filosóficas }\end{array}$ & $\begin{array}{l}\text { Ámbitos conceptuales } \\
\text { y contenidos generales }\end{array}$ \\
\hline $\begin{array}{l}\text { Conciencia } \\
\text { crítica }\end{array}$ & $\begin{array}{l}\text { 1. Distingue los } \\
\text { distintos parámetros } \\
\text { estéticos utilizados } \\
\text { para clasificar una } \\
\text { creación humana } \\
\text { como obra de arte. }\end{array}$ & $\begin{array}{l}\text { 1. ¿Bajo qué } \\
\text { parámetros es } \\
\text { posible otorgar } \\
\text { el estatus de arte } \\
\text { a la obra de un } \\
\text { artista? } \\
\end{array}$ & $\begin{array}{l}\text { 1. La expresión artística } \\
\text { 1.1. El mundo del arte } \\
\text { 1.2. Valor histórico y } \\
\text { cultural del arte }\end{array}$ \\
\hline $\begin{array}{l}\text { Diálogo } \\
\text { interfilosófico }\end{array}$ & $\begin{array}{l}\text { 2. Opina frente } \\
\text { al postulado } \\
\text { posmoderno que } \\
\text { afirma que el arte } \\
\text { ha llegado a su } \\
\text { final, argumentando } \\
\text { en torno a las } \\
\text { consecuencias } \\
\text { artísticas y estéticas } \\
\text { derivadas de dicha } \\
\text { afirmación. }\end{array}$ & $\begin{array}{l}\text { 2. ¿Qué } \\
\text { implicaciones } \\
\text { artísticas y } \\
\text { estéticas tiene } \\
\text { la afirmación } \\
\text { contemporánea } \\
\text { de la muerte del } \\
\text { arte? }\end{array}$ & $\begin{array}{l}\text { 2. Las ideas estéticas } \\
\text { 2.1. Problemas de la } \\
\text { estética } \\
\text { 2.2. La muerte del arte }\end{array}$ \\
\hline
\end{tabular}




\begin{tabular}{|c|c|c|c|}
\hline $\begin{array}{l}\text { Acción } \\
\text { transformadora }\end{array}$ & $\begin{array}{l}\text { 3. Planea estrategias } \\
\text { de acción que } \\
\text { permitan construir } \\
\text { una estética de la } \\
\text { vida cotidiana más } \\
\text { allá de las estéticas } \\
\text { de consumo } \\
\text { dominantes. }\end{array}$ & $\begin{array}{l}\text { 3. ¿Cómo dar } \\
\text { lugar a una } \\
\text { estética de la } \\
\text { vida cotidiana } \\
\text { libre de las } \\
\text { influencias de } \\
\text { la sociedad de } \\
\text { consumo? }\end{array}$ & $\begin{array}{l}\text { 3. Estética y prosaica } \\
\text { de Nuestramérica } \\
\text { 3.1. Cultura estética de } \\
\text { Nuestramérica } \\
\text { 3.2. Prosaica de la } \\
\text { cotidianidad }\end{array}$ \\
\hline $\begin{array}{l}\text { Ciudadanía } \\
\text { intercultural }\end{array}$ & $\begin{array}{l}\text { 4. Manifiesta la } \\
\text { necesidad de unos } \\
\text { valores estéticos que } \\
\text { permitan apreciar las } \\
\text { distintas formas de } \\
\text { expresión artística y } \\
\text { cultural. }\end{array}$ & $\begin{array}{l}\text { 4. ¿Es posible } \\
\text { hablar de un arte } \\
\text { y una estética } \\
\text { de carácter } \\
\text { universal, } \\
\text { o por el } \\
\text { contrario, están } \\
\text { determinados } \\
\text { por su contexto } \\
\text { cultural? }\end{array}$ & $\begin{array}{l}\text { 4. Función social del } \\
\text { arte y decolonialidad } \\
\text { estética } \\
\text { 4.1. Valor del arte } \\
\text { popular } \\
\text { 4.2. Geo-estética } \\
\text { contemporánea }\end{array}$ \\
\hline
\end{tabular}

Nota. Adaptado de: Gaitán y otros (2010). La primera columna: Competencia filosófica, corresponde a las cuatro competencias filosóficas básicas para la filosofía propuestas en las OPFN. La segunda columna: Desempeños, es elaboración propia y describe las cuatro competencias en relación con los núcleos de problemas filosóficos; para esta descripción se utilizó la taxonomía de Bloom revisada y actualizada por Anderson \& Krathwohl (2001). La tercera columna: Preguntas filosóficas, es también elaboración propia y en ella se formulan preguntas problematizadoras en relación con los núcleos de problemas filosóficos y las competencias a desarrollar. La cuarta columna: Ámbitos conceptuales y contenidos generales, sugiere algunos temas y problemas para abordar en cada uno de los cuatro núcleos de problemas filosóficos propuestos.

Partiendo del principio de que la educación es un acto social y humano con responsabilidad ética y política, se plantea por último que las OPFN no son un trabajo de investigación en contra del eurocentrismo o de las culturas del provenientes del Norte, pues por la naturaleza diversa de Nuestramérica el discurso debe ser incluyente y respetuoso, pero si es posible afirmar que constituyen una reflexión crítica en contra de cualquier forma de violencia epistémica con fines hegemónicos, que conlleva necesariamente a la perpetuación de las condiciones coloniales. Dichas orientaciones son también un trabajo en contra de la dominación política y económica que perpetúa el sometimiento de los pueblos y el emplazamiento de la Tierra sustentadora. Finalmente, las OPFN son una propuesta a favor del des- 
silenciamiento cultural que convoca necesariamente al diálogo interfilosófico e intercultural desde la escuela, en búsqueda de un buen vivir para todos en armonía con la Naturaleza.

\section{Referencias}

Apple, M. (2008). Ideología y currículo. Madrid: Ediciones Akal.

Beorlegui, C. (2010). Historia del pensamiento filosófico latinoamericano. Una búsqueda incesante de la identidad. Bilbao: Deusto.

Bustamante, G. (2003). Competencias y evaluaciones masivas en Colombia. Una mirada desde Bordieu. Pedagogía y Saberes, 1(18), pp. 33-44.

Carmona-Cardona, A. (2018). Orientaciones Peodagógicas para la Filosofía desde Nuestramérica. Trabajo de Investigación de Maestria. Universidad de Antioquia, Seccional Oriente. (Sin publicar).

Dussel, E. (1994). Historia de la Filosofía Latinoamericana y Filosofía de la Liberación. Bogotá: Nueva América.

Dussel, E. (2009). Una nueva edad en la historia de la filosofia: el diálogo mundial entre tradiciones filosóficas. Tabula Rasa, 1(11), pp. 97-114.

Freire, P. (2003). El grito manso. Argentina: Siglo XXI.

Freire, P. (2012). Pedagogía de la indignación: Cartas pedagógicas en un mundo revuelto. Buenos Aires: Siglo XXI.

Gaitán, C., López, E., Quintero, M., y Salazar, W. (2010). Documento No. 14: Orientaciones pedagógicas para la filosofía en la Educación Media. Bogotá: MEN. 
Gaos, J. (1944). El pensamiento hispanoamericano. Ciudad de México: El Colegio de México.

ICFES. (2013). Alineación del Examen Saber 11. Bogotá: ICFES.

Gimeno, J. (2008). Diez tesis sobre la aparente utilidad de las competencias en educación. Educarpor competencias, ¿qué hay de nuevo? (pp. 15-58). Madrid: Ediciones Morata.

Mejía, M. R. (2011). Educaciones y pedagogías criticas desde el Sur. Lima: CEAAL.

Melo, S. (2017). Fundamentación conceptual área de filosofía. Bogotá: ICFES.

Ministerio de Educación Nacional. (1994). Ley 115 de 1994.

Ministerio de Educación Nacional. (1996). Resolución 2343 de 1996.

Roig, A. (2004). Teoría y critica del pensamiento latinoamericano. Ciudad de México: Fondo de Cultura Económica.

Torres, J. (1998). El currículum oculto. Madrid: Ediciones Morata. 